\title{
Effectiveness of Metronidazole as Local Drug Delivery in Periodontal Diseases - A Review
}

\author{
Anusha Rajagopalan, Dr. Julie Toby Thomas \\ Saveetha Dental College and Hospital, India
}

\section{Introduction}

Periodontitis refers to gingival inflammation that damages the soft tissue and the supporting structure of the tooth. This further leads to the destruction of alveolar bone surrounding the teeth. Being a multifactorial disease, the factor which plays a role in disease progression is dental plaque. The bacteria adhere to the tooth surface which slowly colonizes and forms bacterial complexes. When the oral hygiene is left unmaintained, it results in gingivitis and these progresses to periodontitis. Periodontitis is thus characterized by destruction of the periodontal ligament, a resorption of the alveolar bone and the migration of the junctional epithelium along the tooth surface. The various periodontal pathogens which are susceptible in the disease progression includes Aggregatibacter (formerly Actinobacillus) actinomycetemcomitans, Porphyromonas gingivalis, Prevotella intermedia, Tannerella forsythia (previously forsythensis), Campylobacter rectus, and Treponema denticola .In the early phase of the disease (gingivitis), inflammation is confined to the gingiva but extends to deeper tissues in periodontitis, leading to gingival swelling, bleeding and bad breath. In the late phase of the disease, the supporting collagen of theperiodontium is degenerated, alveolar bone begins to resorb and gingival epithelium migrates along the tooth surface forming a 'periodontal pocket'.

The main goal of periodontal therapy is the eradication of the plaque bacteria and ensuring the periodontal pockets are free of granulation tissue for proper healing to occur. Mechanical debridement along with some regenerative procedures/ recession management is done for deeper pockets in case of bone defects, but shallow pockets and horizontal bone loss have been managed conservatively by SRP and systemic microbial agents. The value of administering antimicrobial agents as an inexpensive and rapid means of augmenting mechanical periodontal debridement is worth consideration.

Antibiotic therapy has been advocated in periodontal therapy as:

- It can be given as an adjunct to Scaling and Root planning.

- Periodontal maintenance therapy: Recurrent periodontitis usually involves only a few teeth. These sites are ideal for the treatment with this device.

- For medically compromised patients where surgical management is compromised.

- Sustained release device is a less invasive treatment option and it requires less time compared to surgical treatment.

- In cases of severe or aggressive forms of periodontitis in young patients which often cannot be arrested by mechanical treatment alone.

There were many drawbacks in the systemic administration of antibiotics. There was increase risk of antibiotic resistance on prolonged use. Adverse reactions like diarrhea, nausea and pseudo membranous colitis was observed. The side effects including hypersensitivity, gastrointestinal intolerance and the

development of bacterial resistance have been described. Some studies also reported poor results due to the fact that the active product could not achieve an adequate concentration at the site of action and/or due to the inability of the active product to be retained locally for a sufficient period of time.

Hence local drug delivery was introduced so that the drug can be delivered to the local site at a very low concentration compared to systemic drugs with minimal side effects.

Local drug delivery was first proposed by Goodson et al in the year 1979. The periodic use of local delivery systems in reducing probing depths, stabilizing attachment levels and minimizing bleeding would allow better control of the disease.several antibiotics have been incorporated as local drug delivery which can be delivered to the site in periodically

There are two types of local drug delivery - sustained i.e. $<24$ hours and controlled type i.e. $>24$ hours. There are different delivery devices like fibers, gels, films, strips and compacts. 
The antimicrobial drugs available in the different devices are illustrated in table 1. Table 1

\begin{tabular}{|l|l|l|}
\hline Delivery device & Antibiotics available & \multirow{3}{*}{ Sustained /controlled } \\
\hline \multirow{4}{*}{ Fibers } & chlorhexidine & sustained \\
\cline { 2 - 2 } Films & tetracycline & \\
\hline \multirow{4}{*}{ Gels } & tetracycline & sustained \\
\hline \multirow{3}{*}{ Strips } & chlorhexidine hydrochloride & \\
\hline
\end{tabular}

Metronidazole, a nitroimidazole compound which has been proved to be useful as an adjunct after SRP in treating periodontal diseases. It was initially used to treat protozoal infections. It is bactericidal to anaerobic organisms and is believed to disrupt bacterial DNA synthesis in conditions with a low reduction potential. Metronidazole provides high degree of efficacy and relatively few adverse side effects. It is an antibiotic to which susceptible anaerobes have yet to develop clinical resistance. Metronidazole is effective against anaerobes such as Prphyromonas gingivalis and Prevotella intermedia.

The availability of various forms of metronidazole in market: Metronidazole is available in the forms of gels, pastes, tablets, microspheres. Metronidazole $0.75 \%$ topical gel $45 \mathrm{G}$, Metronidazole $0.75 \%$ topical lotion , Microspheres prepared by oil-in-water-oil (O/W/O) double emulsion technique using the blends of poly (D, Llactide-co-glycolide) (PLGA) and polycaprolactone (PCL) in different ratios for the controlled delivery of metronidazole (MTZ). Studies have shown that metronidazole can be released in a sustained manner for a period of one week. This was demonstrated by using a poloxamer 407 gel containing $15 \%$ of metronidazole. This simulated the conditions of humidity, temperature and gingival fluid flow of a periodontal pocket.

Therefore, this article focuses on the effectiveness of metronidazole along with sub-gingival debridement and also highlights various studies where Metronidazole has been used.

\section{Drug Delivery System}

The drug delivered can also be in the form of either natural or synthetic. Synthetic polymers have proved to be extremely interesting because they can be tailor-made to meet pharmacological or biological requirements. Drug delivery systems can be classified according to the mechanism controlling drug release. They are:

(i) Solvent controlled' matrix systems based on macromolecular matrix permeability to small molecules after matrix swelling into hydrated medium;

(ii) Reservoir systems' controlled by drug diffusion across a polymeric membrane;

(iii) Chemically controlled systems' where the rate of drug release is controlled by the rate and extent of degradation of chemical bonds and the erosion of the polymeric matrix. For all these systems, the basic polymer can be of natural origin such as proteins7or collagen, semi-synthetic such as cellulose derivative, or synthetic, all of which must preferably degrade during use. Natural polymers have been considered as biodegradable carriers. However; most of them have disadvantages inherent to their structure, including limited half-life, complexity of composition and immunogenicity due to the polymer itself or to its degradation by-products. Some of these systems are not resorbable, while most are biodegradable. Non biodegradable systems have to be removed after complete drug release, which may cause irritation and inflammation of the treated site. Intra pocket devices can be divided in two broad categories depending on degradability. Nondegradable

Devices (first generation) and degradable devices (second generation).Non degradable devices have the advantage that the therapist controls the removal of the device and therefore has greater control over the time of exposure of the pocket environment to the drug.

Together with the solid devices, semisolid formulations also receive reasonable attention for the localized delivery of antibiotics. Semisolid or gel formulations can indeed have some advantages. They possess a higher biocompatibility and bioadhesivity, allowing adhesion to the mucosa in the dental pocket and, finally, they can be rapidly eliminated through normal catabolic pathways, decreasing the risk of irritative or allergic host reactions at the application site. Various oleogels and hydrogels for the delivery of tetracycline $(2.5 \%)$, metronidazole $(25 \%)$, metronidazole benzoate $(40 \%)$, as well as a combination of tetracycline $(2.5 \%)$ and 
metronidazole benzoate (40\%), have been tested and satisfactory results have been achieved. The gels composed of cellulose derivatives such as hydroxypropylmethyl cellulose.

Bioadhesion or mucoadhesion is a preliminary requirement for prolonged release of the drug at the site. The retention time, as determined by fluorescein release, was found to be significantly higher for chitosan gel as compared to xanthan gum and poly(- ethylene oxide) gel. Chitosan, a novel biodegradable natural polymer, in a gel form $(1 \%, w / w)$ with or without $15 \%$ metronidazole, had demonstrated effectiveness in the treatment of chronic periodontitis.

These systems exhibit a pseudoplastic flow and thermoresponsive behaviour, existing as a liquid at room temperature and gel at 34-37 degree $\mathrm{C}$.

\section{Clinical Trials Using Metronidazole Gels}

In the year 1999, Kinane conducted a six-month comparison of three periodontal local antimicrobial therapies in persistent periodontal pockets. The results showed the mean probing depth reductions. In case of scaling +tetracycline, it was found to be $1.38 \mathrm{~mm}$; in scaling +metronidazole, it was found to be $0.93 \mathrm{~mm}$; in scaling +minocycline, it was found to be $1.10 \mathrm{~mm}$ and scaling alone was found to be $0.71 \mathrm{~mm}$. thus, it was found that scaling plus an adjunct in the form of antimicrobial system was found more effective.

In the year 2000, Pedrazzoli conducted a study on comparing the clinical and microbiological effects of topical subgingival application of metronidazole $25 \%$ dental gel and scaling in the treatment of adult periodontitis. The study showed that Local metronidazole treatment induced a significant and long-lasting shift in the subgingival flora towards a composition more compatible with health and comparable to that obtained by mechanical debridement. Proportions of black pigmented anaerobes including Prevotella intermedia, and the number of spirochetes were significantly reduced after treatment with a concomitant increase in the proportions of streptococci. While scaling resulted in a statistically significant increase in the proportion of A. actinomycetemcomitans, this was avoided after metronidazole treatment.

In the year 2001, Yang H conducted a study to evaluate the clinical effects of metronidazole ofloxacin gel (MO gel) as an adjunct to conventional therapy for periodontitis. The study indicates local application with MO gel treatment as an adjunct to conventional therapy may lead to a more advantageous outcome for periodontitis.

In the year 2005, Marija Toskić-Radojičić* conducted a study on the effects of topical applicaton of metronidazole -containing mucoadhesive lipogel in periodontal pocket. The study showed that Metronidazole, in a lipogel-type base applied in the concentration of $25 \%$, provided an efficient treatment of anaerobic infection in the periodontal pockets.

In the year 2009, Singh S conducted a study on evaluation of two local drug delivery systems as adjuncts to mechanotherapy as compared to mechanotherapy alone in management of chronic periodontitis. In this study there were three groups that were labeled as group A (Scaling + Tetracycline), group B (Scaling + Metronidazole), and group C (Scaling alone). A microbiological analysis was carried out to determine the efficacy of these systems in changing the pathogenic flora in deep pockets. In addition, a multiplex polymerase chain reaction was carried out to confirm the presence of Actinobacillus actinomycetemcomitans, Porphyromonas gingivalis $(\mathrm{Pg})$, and Tannerella forsythensis in the flora associated with chronic periodontitis. The study showed that there was clinical improvement in groups A and B, which correlated with an improvement in the microbiological parameters; these results were sustained for 90 days following therapy. In Group C, the flora showed a shift towards baseline at the end of 90 days. both the local antibiotic therapies resulted in greater improvement in microbiological parameters when used as an adjunct to mechanotherapy as compared to mechanotherapy alone.Both the local antibiotic therapies resulted in greater improvement in microbiological parameters when used as an adjunct to mechanotherapy as compared to mechanotherapy alone.

\section{Metronidazole Loaded Bio-Absorbable Film}

In the year 2002, Kurtis B et al conducted an in vitro study to evaluate the regenerative potential of a metronidazole-loaded biodegradable (polylactide/glycolide) (PLGA) GTR membrane.the study showed that PLGA membranes with and without metronidazole have a beneficial effect on periodontal regeneration.

In the year 2009, Shifrovitch Y et al did a study on treatment of infected periodontal pockets using Metronidazole-loaded bioabsorbable films as local antibacterial agent. The study enabled the understanding of metronidazole-release kinetics from bioabsorbable polymeric films. The developed systems demonstrated good biocompatibility and the ability to inhibit Bacteroides fragilis growth; therefore, they may be useful in the treatment of periodontal diseases.

\section{Metronidazole Nanofibers}

A new sustained release drug system of poly e-caprolactone (PCL) nanofibers containing metronidazole (MET) was successfully electrospun and evaluated clinically for periodontal diseases. 
In the year 2012, Chaturvedi et al conducted a clinical study on evaluation of metronidazole nanofibers in patients with chronic periodontitis. It was found that Combination of SRP + MET nanofibers resulted in added benefits and was more effective when compared to the control group which was treated only with scaling and root planning.

In the year 2012 , Reise et al conducted a study to achieve detailed biomaterials characterization of a drug delivery system for local periodontitis treatment based on electrospun metronidazole-loaded resorbable polylactide (PLA) fibers. This study shows that Metronidazole-loaded electrospun fiber mats represent an interesting class of resorbable drug delivery systems. Sustained drug release properties and cytocompatibility suggest their potential clinical applicability for the treatment of periodontal diseases.

\section{Recent advances in local drug delivery systems}

Vesicular liposomal systems are designed to mimic the bio-membranes in terms of structure and biobehaviour, and hence are investigated intensively for targeting periodontal biofilms. Succinylated ConcanavalinA (lectin)-bearing liposomes (proteoliposomes) have been found to be effective for the delivery of triclosan to periodontal biofilms. Jones and Kaszuba reported interactions between liposomes made up of phosphatidylinositol (PI) and bacterial biofilms.

The nanoparticle system provides several advantages as compared with microspheres, micro particles and emulsion-based delivery systems, including high dispersibility in an aqueous medium, controlled release rate and increased stability. Biocompatible nanoparticles composed of 2-hydroxyethyl methacrylate (HEMA) and polyethylene glycol dimethacrylate (PEGDMA) could be used as a drug delivery system for dental applications.

\section{Conclusion}

Periodontal disease having a multifactorial etiology, where periodontal pathogens mainly include anaerobic organisms can be eradicated by mechanical debridement and antimicrobial therapy. Systemic administration of antimicrobials have faced major drawbacks like increase risk of antibiotic resistance on prolonged use. Adverse reactions like diarrhea, nausea and pseudo membranous colitis was also observed. Hence, local drug delivery were formulated which can deliver the drug to appropriate site. Metronidazole being effective in treatment of anaerobic infections can be formulated in local drug devices. Hence, further studies have been warranted to demonstrate the efficacy of the drug. 